\title{
400 pc Imaging of a Massive Quasar Host Galaxy at a Redshift of 6.6
}

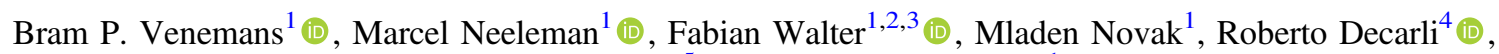 \\ Joseph F. Hennawi ${ }^{5}$ (D), and Hans-Walter Rix $^{1}$ (iD) \\ ${ }^{1}$ Max-Planck Institute for Astronomy, Königstuhl 17, D-69117 Heidelberg, Germany; venemans@mpia.de \\ ${ }^{2}$ Astronomy Department, California Institute of Technology, MC105-24, Pasadena, CA 91125, USA \\ ${ }^{3}$ National Radio Astronomy Observatory, Pete V. Domenici Array Science Center, P.O. Box 0, Socorro, NM 87801, USA \\ ${ }_{5}^{4}$ Osservatorio di Astrofisica e Scienza dello Spazio di Bologna, via Gobetti 93/3, I-40129 Bologna, Italy \\ ${ }^{5}$ Department of Physics, Broida Hall, University of California, Santa Barbara, CA 93106-9530, USA \\ Received 2019 February 19; revised 2019 March 20; accepted 2019 March 20; published 2019 April 5
}

\begin{abstract}
We report high spatial resolution $\left(\sim 0 !^{\prime \prime} 076,410 \mathrm{pc}\right)$ Atacama Large Millimeter/submillimeter Array imaging of the dust continuum and the ionized carbon line [C II] in a luminous quasar host galaxy at $z=6.6,800$ million years after the big bang. Based on previous studies, this galaxy hosts a $\sim 1 \times 10^{9} M_{\odot}$ black hole and has a star formation rate of $\sim 1500 M_{\odot} \mathrm{yr}^{-1}$. The unprecedented high resolution of the observations reveals a complex morphology of gas within $3 \mathrm{kpc}$ of the accreting central black hole. The gas has a high velocity dispersion with little ordered motion along the line of sight, as would be expected from gas accretion that has yet to settle in a disk. In addition, we find the presence of [C II] cavities in the gas distribution (with diameters of $\sim 0.5 \mathrm{kpc}$ ), offset from the central black hole. This unique distribution and kinematics cannot be explained by a simple model. Plausible scenarios are that the gas is located in a truncated or warped disk, or the holes are created by interactions with nearby galaxies or due to energy injection into the gas. In the latter case, the energy required to form the cavities must originate from the central active galactic nucleus, as the required energy far exceeds the energy output expected from supernovae. This energy input into the gas, however, does not inhibit the high rate of star formation. Both star formation and black hole activity could have been triggered by interactions with satellite galaxies; our data reveal three additional companions detected in [C II] emission around the quasar.
\end{abstract}

Key words: cosmology: observations - galaxies: active - galaxies: high-redshift - galaxies: ISM - galaxies: star formation - quasars: individual (VIK J030516.92-315056)

\section{Introduction}

It is expected that massive black holes in the early universe $(z \gg 6)$ are located in nascent massive galaxies (e.g., Valiante et al. 2014; Di Matteo et al. 2017). At these high redshifts, supermassive black holes are not detected directly, but their presence is inferred as they power luminous quasars through accretion. Such luminous quasars have been found up to $z=7.5$ (Bañados et al. 2018), indicating that supermassive black holes were already formed at $z>7$ (e.g., Mortlock et al. 2011; De Rosa et al. 2014; Mazzucchelli et al. 2017; Bañados et al. 2018).

Using (sub-)millimeter facilities, such as the Atacama Large Millimeter/submillimeter Array (ALMA) and the IRAM Northern Extended Millimeter Array, the interstellar medium (ISM) of $z>6$ quasar host galaxies have now been routinely detected (e.g., Bertoldi et al. 2003; Walter et al. 2003, 2004; Maiolino et al. 2005; Wang et al. 2010, 2013; Venemans et al. 2012, 2016, 2018; Willott et al. 2013, 2015; Decarli et al. 2018). While the global properties of the quasar hosts are now reasonably well determined (e.g., Decarli et al. 2018; Venemans et al. 2018), most observations barely resolve the galaxies (i.e., apparent galaxy size $\lesssim 2$ times the size of the resolution of the observations). The outstanding imaging capabilities of ALMA now allow us to investigate the ISM in quasar host galaxies well below kiloparsec scales, and study the distribution and kinematics of gas and dust in extraordinary detail.

The quasar J0305-3150 at $z=6.6$ was discovered in 2013 (Venemans et al. 2013) and has a black hole mass of $\sim 1 \times 10^{9}$ $M_{\odot}$ (Venemans et al. 2013; De Rosa et al. 2014; Mazzucchelli et al. 2017). Previous ALMA observations at 0."62 (3.4 kpc at $z=6.6)$ resolution of the [C II $] 158 \mu \mathrm{m}$ emission line and the underlying dust continuum (Venemans et al. 2016) revealed that the black hole is hosted by an ultraluminous infrared host galaxy (far-infrared (FIR) luminosity $L_{\mathrm{FIR}}$ exceeding $10^{12} L_{\odot}$ ). Based on the dust continuum and the detection of $\mathrm{CO}(6-5)$ and $\mathrm{CO}(7-6)$ (Venemans et al. 2017), the estimated molecular gas mass in J0305-3150 is $(2.4-18) \times 10^{10} M_{\odot}$. This gas mass is $>60 \%$ of the dynamical mass derived from the extent and width of the [C II] line (Venemans et al. 2016), which has an FWHM of $255 \mathrm{~km} \mathrm{~s}^{-1}$. These earlier observations indicated that the [C II] emission line spectrum shows nonvirial motion, consistent with the presence of an outflow or companion galaxy.

Here we present high spatial resolution (0".076) ALMA observations of the host galaxy of quasar J0305-3150. The Letter is organized as follows. In Section 2 we provide details of the new observations. The results are discussed in Section 3. We present the spatial distribution of the gas and dust in the quasar host in Section 3.1 and we investigate the gas kinematics in Section 3.2. In Section 4 we introduce a model to understand the observed gas kinematics. The constraints from the model are presented in Section 4.1. In Section 5 we explore alternative scenarios to explain the cavities seen in the gas distribution and introduce the presence of companion [C II] emitters in the field in Section 6. We conclude with a summary in Section 7.

Throughout this Letter we adopt a Lambda cold dark-matter cosmology with a Hubble constant of $H_{0}=70 \mathrm{~km} \mathrm{~s}^{-1} \mathrm{Mpc}^{-1}$, a mass density of $\Omega_{M}=0.3$, and a vacuum density of $\Omega_{\Lambda}=0.7$, which is consistent with the latest Planck 
measurements (Planck Collaboration et al. 2016). With these cosmological parameters, the age of the universe at $z=6.6$ is 810 Myr. Far-infrared luminosities, $L_{\mathrm{FIR}}$, are computed by integrating the dust spectral energy distribution (SED) between the rest-frame wavelengths 42.5 and $122.5 \mu \mathrm{m}$ (e.g., Helou et al. 1988). For the shape of the dust SED we follow the literature and assume a dust temperature of $47 \mathrm{~K}$ and a dust emissivity index of $\beta=1.6$ (e.g., Beelen et al. 2006; Wang et al. 2008, 2013; Willott et al. 2013; Venemans et al. 2016). Uncertainties in the FIR luminosity quoted in this Letter represent measurement uncertainties only. The uncertainties introduced in $L_{\mathrm{FIR}}$ due to the unknown shape of the dust SED are a factor of 2-3 (see the extensive discussion in Venemans et al. 2018).

\section{ALMA Observations}

To resolve the structure of the host galaxy and explore its detailed kinematics, we observed J0305-3150 between 2017 November 12 and 18 with ALMA in configuration C43-8. The number of antennas was 43 with baselines between 92 and $13,894 \mathrm{~m}$. The ALMA observations covered the redshifted [C II] line observed at $249.6 \mathrm{GHz}$ with a single bandpass with a frequency width of $1.875 \mathrm{GHz}\left(\sim 2250 \mathrm{~km} \mathrm{~s}^{-1}\right)$. Three other bandpasses of $1.875 \mathrm{GHz}$ each were placed to measure the FIR continuum at observed frequencies of 252.1, 264.6, and 266.6 GHz. Bandpass and flux calibration was performed through observations of J0522-3627. For the phase calibration, the source J0326-3243 was observed. The total observing time was $7.6 \mathrm{hr}$, of which $3.5 \mathrm{hr}$ were on-source.

The data were reduced using the Common Astronomy Software Application package (McMullin et al. 2007), following the standard reduction steps. The rms noise around the redshifted [C II] line is $87 \mu \mathrm{Jy}_{\text {beam }}^{-1}$ per $30 \mathrm{MHz}$ bin $\left(36 \mathrm{~km} \mathrm{~s}^{-1}\right)$. The final beam using natural weighting has a size of 0 " $076 \times 0$ ". 071 , which corresponds to $410 \mathrm{pc} \times 385 \mathrm{pc}$ at $z=6.6$. With this resolution, we can resolve structures on scales of $\sim 400 \mathrm{pc}$ in the quasar host galaxy and look for possible interplay between the accreting black hole and its host. For a black hole mass of $\sim 10^{9} M_{\odot}$ and with the gas having a velocity dispersion of $\sim 110 \mathrm{~km} \mathrm{~s}^{-1}$ (based on the FWHM of $255 \mathrm{~km} \mathrm{~s}^{-1}$ ), the region where the black hole dominates the gravitational potential, the so-called black hole sphere of influence, has a radius of $\sim 355 \mathrm{pc}$. Thus the achieved spatial resolution of the observations in principle allow us to probe scales similar to the black hole sphere of influence in order to search for kinematic signatures of the central black hole.

\section{Results}

\subsection{Dust and [C II] Emission}

In Figure 1 we show the results of the observations. The first two panels in the figure show the $[\mathrm{C}$ II] and continuum maps. The bottom two panels in Figure 1 show the kinematics of the [C II] emission. To match the position of the [C II] and FIR dust emission with that of the accreting black hole, we recomputed the near-infrared (NIR) location of the quasar by correcting the positions of nearby stars with a match in the GAIA DR2 catalog (Gaia Collaboration et al. 2018). The resulting NIR location of the quasar is $03^{\mathrm{h}} 05^{\mathrm{m}} 16^{\mathrm{s}} 919,-31^{\circ} 50^{\prime} 55^{\prime \prime} .86$ with an uncertainty of 0 !" 02 , which is consistent with the previously published coordinates of the quasar (Venemans et al. 2013, 2016). The distribution of the FIR continuum emission has a pronounced peak that is coincident with the NIR location of the quasar.

We measured the total flux of the quasar host galaxy in an aperture with a radius of 0.75 . The total continuum flux density is $5.34 \pm 0.19 \mathrm{mJy}$ around the [C II] line, making this quasar host one of the most luminous, unlensed sources known at high redshift (e.g., Venemans et al. 2018). The total brightness of the [C II] line is $5.43 \pm 0.33 \mathrm{Jy} \mathrm{km} \mathrm{s}^{-1}$, which corresponds to a luminosity of $L_{[\mathrm{C} \text { II }]}=(5.90 \pm 0.36) \times 10^{9}$ $L_{\odot}$. We derive a systemic redshift of the quasar host by fitting a Gaussian to the [C II] emission line, resulting in $z_{[\mathrm{C} \mathrm{II}]}=6.61391 \pm 0.00015$, consistent with earlier measurements (Venemans et al. 2016).

Both the continuum and the [C II] emission are spatially resolved, and the emission is extended over $\sim 5 \mathrm{kpc}$. The gas distribution and kinematics, as traced by the [C II] emission, are highly complex. There is a pronounced lack of [C II] emission toward the east of the quasar. This cavity in the [C II] emission is also seen in the dust continuum observations. In general, the continuum and [C II] emission trace similar structures, the main difference being the bright peak in the continuum.

In Figure 2 we compare the [C II] emission to that of the dust. The $L_{[\mathrm{C} \mathrm{II]}} / L_{\mathrm{FIR}}$ ratio is lowest at the location of the quasar where the dust continuum peaks $\left(L_{[\mathrm{C} \text { II }]} / L_{\mathrm{FIR}} \approx 1.5 \times 10^{-4}\right)$. This is reminiscent of the centers of local star bursts and ultraluminous infrared galaxies, where low [C II]-to-FIR luminosity ratios are observed in regions with high FIR surface brightness (e.g., Smith et al. 2017; Herrera-Camus et al. 2018). Away from the central regions, the [C II]-to-FIR luminosity ratio is in the range of $L_{[\mathrm{C} \text { II }]} / L_{\mathrm{FIR}}=(0.5-1) \times 10^{-3}$. More centrally concentrated continuum emission has been observed in some other high-redshift galaxies and quasar hosts, albeit at lower spatial resolution (e.g., Wang et al. 2013; Capak et al. 2015; Venemans et al. 2016; Gullberg et al. 2018).

\subsection{Gas Dynamics}

From the mean velocity map of the [C II] emission (third panel in Figure 1) it is evident that the position of the accreting black hole coincides with the kinematic center of the [C II] emission. It is also located at the center of the global [C II] emission. Generally, the gas exhibits some ordered motion along the line of sight, with the gas having positive line-of-sight velocities toward the east and negative velocities toward the west. The highest velocity component, which was already discovered in low-resolution data (Venemans et al. 2016), is now clearly separated, both spatially and in frequency. It is therefore likely that this is a companion galaxy close to the quasar host (see also Figure 3 and 6). This will be further discussed in Section 6.

The velocity dispersion in the quasar host is roughly uniform (in the range $50-100 \mathrm{~km} \mathrm{~s}^{-1}$ ) throughout the galaxy. The dispersion is almost equal to the projected line-of-sight velocity. At the position of the quasar, there is no sharp increase in the velocity dispersion; the dispersion of $110 \mathrm{~km} \mathrm{~s}^{-1}$ at that location is not found to be higher compared to the remainder of the host galaxy. This indicates that, with the current resolution, the mass budget within the central $\sim 400 \mathrm{pc}$ is not dominated by the black hole. Indeed, assuming typical dust properties (dust temperature $T_{d}=47 \mathrm{~K}$ and an emissivity index $\beta=1.6$; e.g., Beelen et al. 2006) and a gas-to-dust ratio of 70 (e.g., Sandstrom et al. 2013), the inferred gas mass within our central resolution element is $6 \times 10^{9} M_{\odot}$ (with significant 


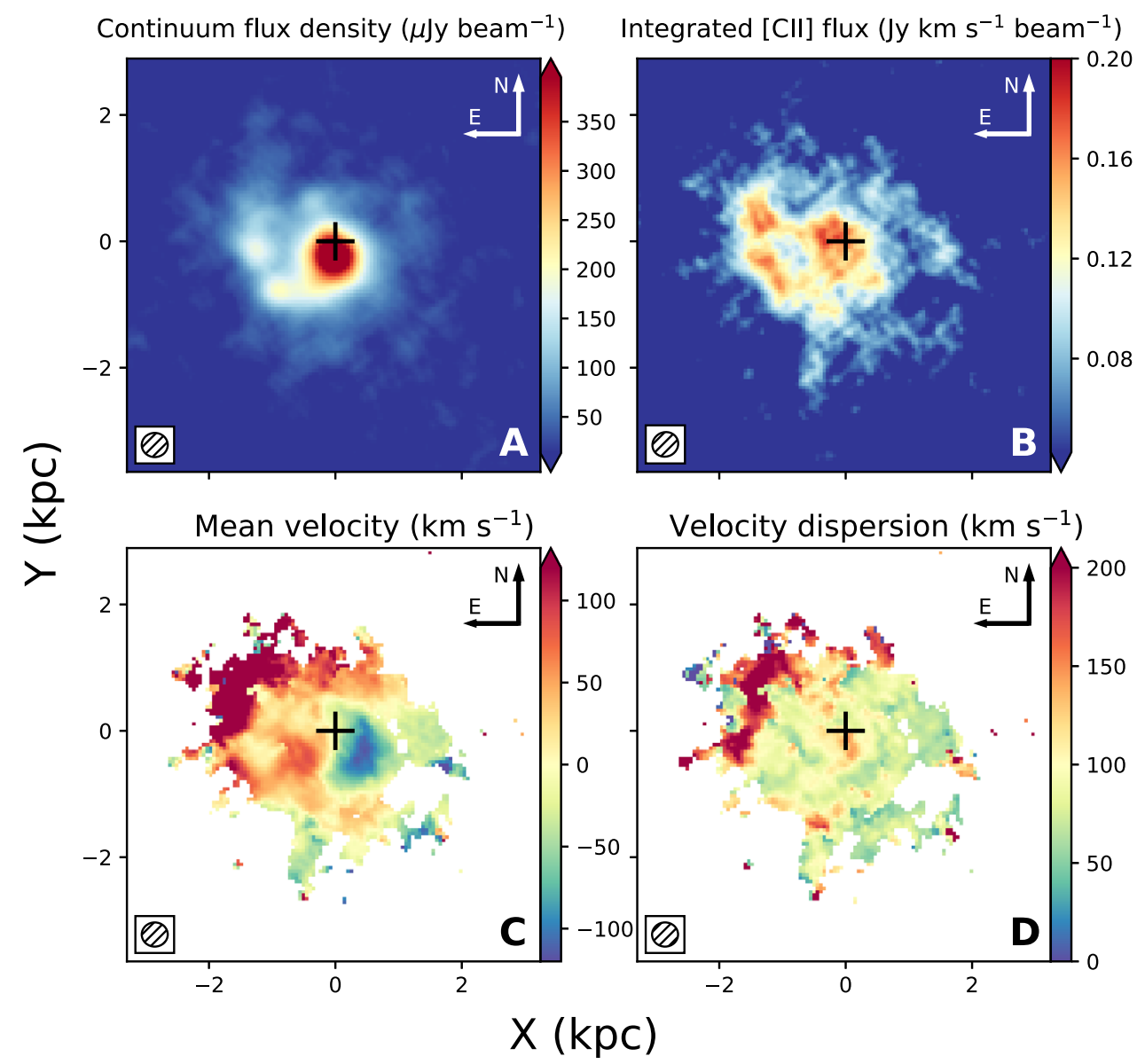

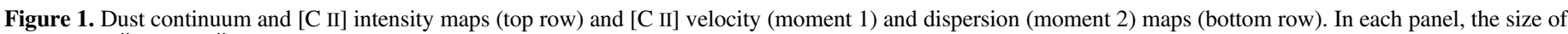

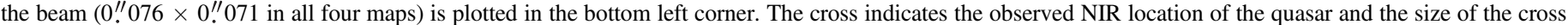

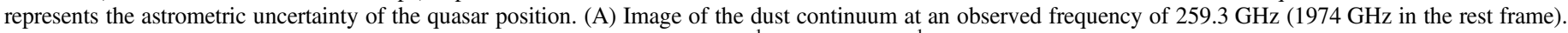

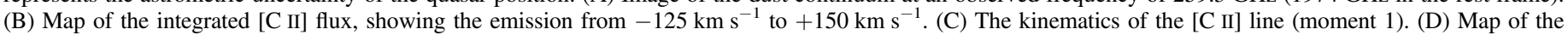
velocity dispersion of the $[\mathrm{C} \mathrm{II}]$ emission (moment 2).

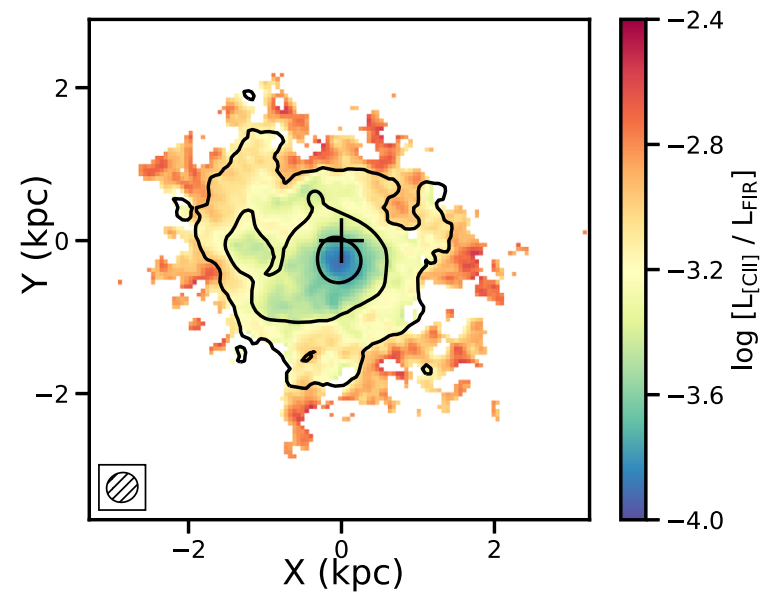

Figure 2. Map of the [C II]-to-FIR luminosity ratio. The $L_{[\mathrm{C} \mathrm{II}]} / L_{\mathrm{FIR}}$ ratio is plotted in color, and the contours represent the dust emission at $6 \sigma, 18 \sigma$, and $54 \sigma$ levels (see also Figure 1 ). The lowest values in $L_{[\mathrm{C} \mathrm{II}]} / L_{\mathrm{FIR}}$ coincide with the peak of the dust continuum emission and the position of the quasar.

uncertainties; see, e.g., the discussion in Venemans et al. 2018), higher than the $10^{9} M_{\odot}$ of the black hole (De Rosa et al. 2014).

To explore how the distribution of [C II] emission changes with line-of-sight velocity, we have averaged the emission into channels with a width of $30 \mathrm{MHz}\left(36 \mathrm{~km} \mathrm{~s}^{-1}\right.$; see Figure 3).
There are two striking features in the channels centered around $0 \mathrm{~km} \mathrm{~s}^{-1}$ : (i) the [C II] emission covers the whole spatial extent seen in the integrated emission map (Figure 1), and (ii) there are two depressions/cavities in the [C II] emission with diameters of $\sim 0.5 \mathrm{kpc}$ on either side of the black hole. The gas with the highest velocities - that was already seen at positive velocities toward the northeast in the low-resolution data (Venemans et al. 2016) - is clearly offset from the quasar host.

We can capture some of the complexity of the kinematics by generating a position-velocity diagram of the [C II] emission (Figure 4). A position-velocity diagram is a planar slice through the data cube and a useful way to visualize the complex kinematics as it shows the line-of-sight velocities of the gas as a function of distance from the center. This pseudolongslit spectrum, oriented along the major kinematical axis through the position of the quasar and the [C II] cavity to the east, shows an S-like shape in the velocity curve. This implies that, in addition to the cavity that is clearly seen toward the east, there is a second cavity to the west, which is also seen in the channel maps at zero velocity (Figure 3). Interestingly, the line-of-sight velocities approach zero at the largest radii, consistent with the finding that the channel map at zero velocity shows the full extent of the integrated line emission. 


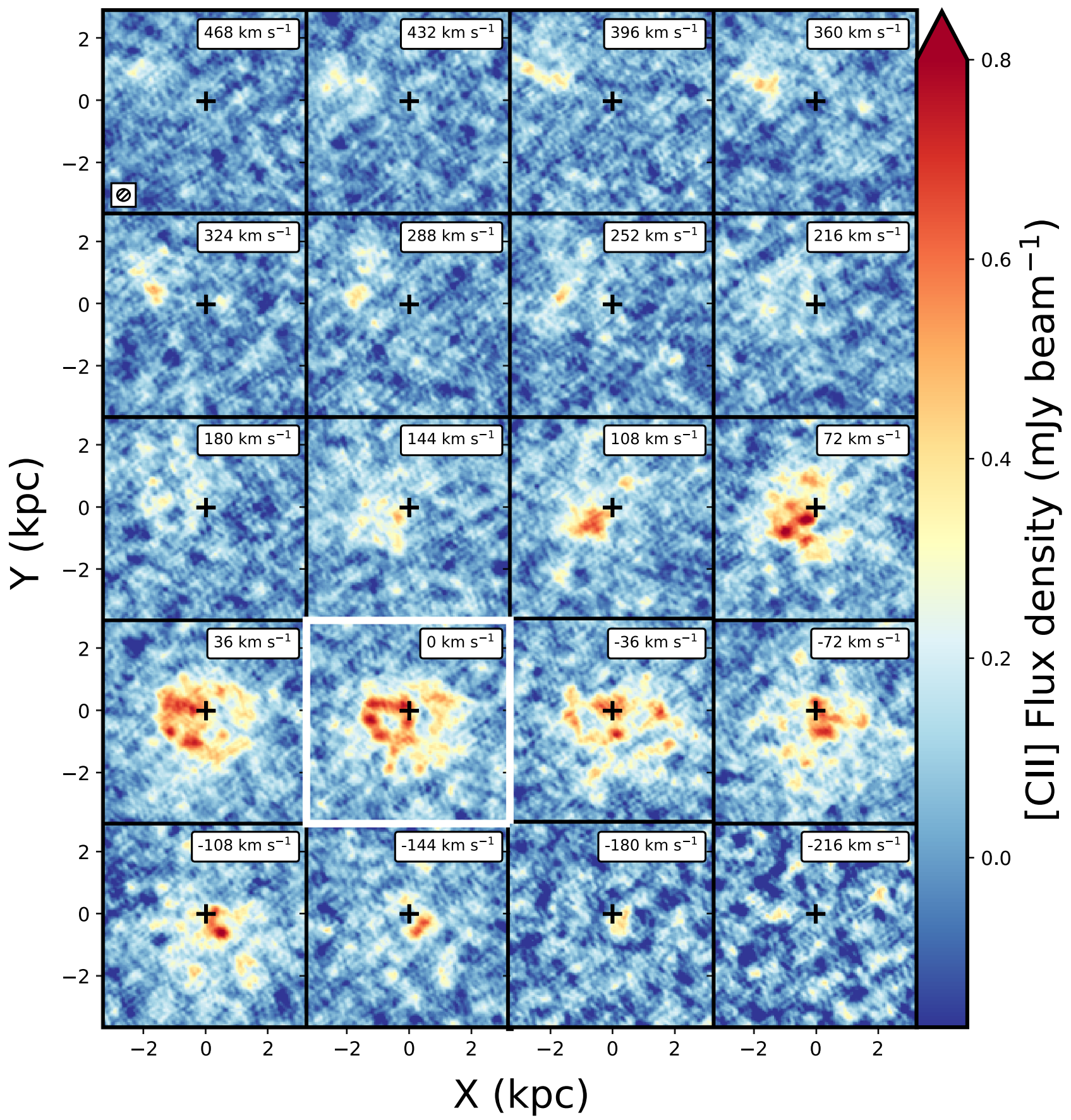

Figure 3. Channel maps of the $[\mathrm{C} \mathrm{II}]$ emission. For each image the emission was averaged over $30 \mathrm{MHz}\left(36 \mathrm{~km} \mathrm{~s}^{-1}\right)$. The cross indicates the location of the quasar (here defined as the position of the peak of the FIR continuum; see Section 3.1). At the top of each image the average velocity (compared to the systemic redshift of $z=6.61391)$ is indicated

In the next section, we will fit several models to explain the distribution and velocity of the gas.

\section{Kinematic Modeling of the $[\mathrm{C}$ II $]$ Emission}

To better understand the spatial extent and kinematic signature of the $[\mathrm{CII}]$ emission, we have fitted a range of simple models to the data. These models are discussed below and are summarized in Table 1. To fit the models, we generate a cube from the given model parameters, which we will refer to as the model cube. This model cube is then convolved with the ALMA primary beam to account for potential beam smearing effects. We compare this convolved model data cube, $M$, with the data cube, $D$, using a standard chi-squared algorithm,

$$
\chi^{2}=\sum_{i}\left(M_{i}-D_{i}\right)^{2} / U_{i}^{2},
$$

where $U_{i}$ is the uncertainty (i.e., rms uncertainty of the pixels showing no emission in the same channel as pixel $i$ ). The model cube has velocities that range from -216 to $+216 \mathrm{~km} \mathrm{~s}^{-1}$. This range covers the velocities where the data show [C II] emission but excludes the channels affected by the close companion (see Figures 3 and 6). Since the adjacent pixels are highly correlated in interferometric data, we do not sum up all of the pixels in our data, but instead use a bootstrap 

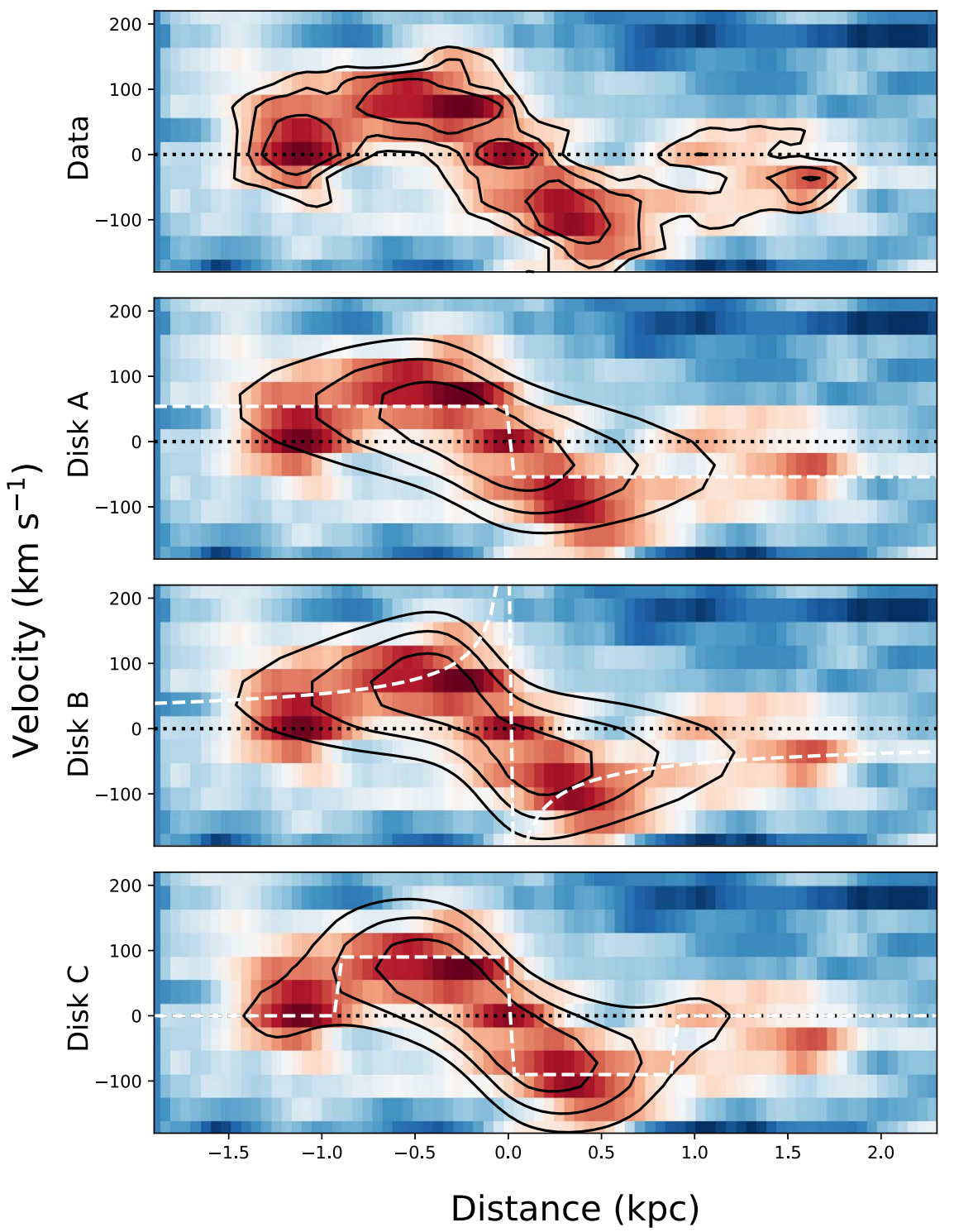

Figure 4. Position-velocity diagram along the major axis, illustrating how the velocity of the gas changes as a function of distance from the black hole. The major axis is aligned approximately east to west. In each panel, the color image represents the data. In the bottom three panels, the black contours show the fit to the data of three different models. These models are described in detail in Section 4.1.

method, whereby we randomly select $N$ pixels from the data cube and calculate the $\chi^{2}$ value of these pixels. We repeat this process until the median $\chi^{2}$ value remains unchanged. Here the number of pixels, $N$, is chosen so that on average each beam only contains a single pixel per single $\chi^{2}$ calculation.

To fully sample the parameter space of the model and provide more realistic constraints on the possible values of each parameter, we have used a Markov chain Monte Carlo (MCMC) method to sample the parameter space. In particular, we have used the affine invariant MCMC ensemble sampler code, emcee (Foreman-Mackey et al. 2013). For all parameters, we assume flat priors, and we initiate the parameters with rough estimates from visual inspection of the data. We have verified that these initial guesses do not affect the results of the MCMC analysis. Results of the different models are shown in Figure 5 and Table 1.

\subsection{Modeling Results}

We have modeled the [C II] kinematics in 3D using several simple models. The following models were considered in this analysis: (a) a thin rotating disk with a constant velocity profile (Disk A). This is the fiducial profile of a dark-matter-dominated disk galaxy, which normally is the model employed to explain the gas kinematics in marginally resolved $z \sim 6$ quasar host galaxies (e.g., Wang et al. 2013; Venemans et al. 2016; Shao et al. 2017). Model (b) is a thin rotating disk with a decreasing velocity profile (Disk B). This profile would arise if the mass of the galaxy is centrally located. Model (c) is a thin rotating disk with a constant velocity profile up to a certain radius, after which it decreases to zero (Disk C). This is the velocity profile of a disk that is truncated at a certain radius, or the velocity profile of a disk that is warped into the plane of the sky at this radius. Model (d) is an inclined biconical jet embedded in a uniform rotating spherical gas (Rot + Jet $D)$. This is a simple model of an active galactic nucleus (AGN) jet accelerating and/or ionizing the [C II] emitting ISM. This possibility will be 
Table 1

Model Parameters

\begin{tabular}{|c|c|c|c|c|}
\hline & Disk A & Disk B & Disk C & Rot + Jet $\mathrm{D}$ \\
\hline Velocity profile & Constant & Keplerian & Truncated & Constant \\
\hline R.A. ${ }^{\mathrm{a}, \mathrm{b}}$ & $03^{\mathrm{h}} 05^{\mathrm{m}} 16^{\mathrm{s}} \cdot 9225(1)$ & $03^{\mathrm{h}} 05^{\mathrm{m}} 16^{\mathrm{s}} .9219(1)$ & $03^{\mathrm{h}} 05^{\mathrm{m}} 16^{\mathrm{s}} .9219(1)$ & $03^{\mathrm{h}} 05^{\mathrm{m}} 16^{\mathrm{s}} .9227(2)$ \\
\hline decl. $^{\mathrm{a}, \mathrm{b}}$ & $-31^{\circ} 50^{\prime} 55^{\prime \prime} 938(2)$ & $-31^{\circ} 50^{\prime} 55^{\prime \prime} 939(2)$ & $-31^{\circ} 50^{\prime} 55^{\prime \prime} 940(1)$ & $-31^{\circ} 50^{\prime} 55^{\prime \prime} 939(3)$ \\
\hline Redshift $^{\mathrm{a}, \mathrm{b}}$ & $6.61405(3)$ & $6.61396(3)$ & $6.61388(3)$ & 6.61404(3) \\
\hline Inclination (deg.) & $38.0_{-2.8}^{+2.0}$ & $38.5_{-2.0}^{+1.6}$ & $34.7_{-1.7}^{+1.8}$ & $\ldots$ \\
\hline Position angle (deg.) & $257_{-1.4}^{+1.4}$ & $262_{-1.3}^{+1.2}$ & $271_{-1.1}^{+1.2}$ & $249_{-2.0}^{+1.9}$ \\
\hline Central intensity $\left(\mathrm{mJy}\right.$ beam ${ }^{-1}$ ) & $0.78_{-0.12}^{+0.11}$ & $0.85_{-0.11}^{+0.11}$ & $0.86_{-0.12}^{+0.13}$ & $0.81_{-0.31}^{+0.33}$ \\
\hline Intensity scale radius $(\mathrm{kpc})$ & $1.41_{-0.03}^{+0.03}$ & $1.34_{-0.03}^{+0.03}$ & $1.29_{-0.02}^{+0.03}$ & $0.91_{-0.02}^{+0.02}$ \\
\hline Circular velocity $\left(\mathrm{km} \mathrm{s}^{-1}\right)$ & $87_{-4}^{+6}$ & $96_{-18}^{+23}$ & $157_{-9}^{+8}$ & $103_{-5}^{+9}$ \\
\hline Velocity dispersion $\left(\mathrm{km} \mathrm{s}^{-1}\right)$ & $99.3_{-0.9}^{+0.9}$ & $95.6_{-0.98}^{+1.0}$ & $94.6_{-1.0}^{+0.98}$ & $99.7_{-1.2}^{+1.1}$ \\
\hline Velocity scale radius (kpc) & $\ldots$ & $0.81_{-0.28}^{+0.39}$ & $0.98_{-0.01}^{+0.04}$ & $\cdots$ \\
\hline Jet opening angle (deg.) & $\cdots$ & $\ldots$ & $\ldots$ & $28.6_{-2.9}^{+2.7}$ \\
\hline Jet $z$-axis angle (deg.) ${ }^{c}$ & $\cdots$ & $\cdots$ & $\cdots$ & $37.8_{-2.9}^{+2.7}$ \\
\hline Jet $x$-axis angle (deg. $)^{\mathrm{d}}$ & $\ldots$ & $\ldots$ & $\ldots$ & $-135_{-7}^{+7}$ \\
\hline
\end{tabular}

Notes.

${ }^{\text {a }}$ Position of the kinematic center of the model.

${ }^{\mathrm{b}}$ Number in parentheses is the uncertainty in the last number.

${ }^{c}$ Angle of the jet compared to the axis of rotation.

d Angle of the jet compared to the position angle of the rotation.
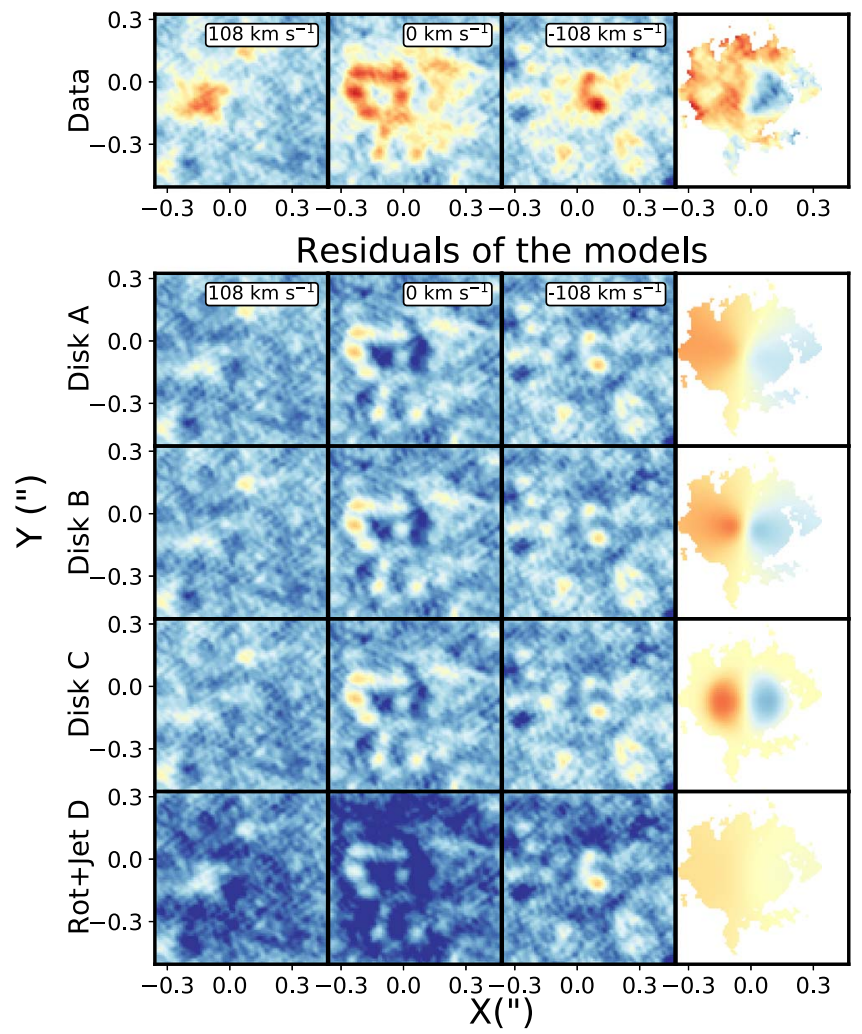

Figure 5. Figure of the different models. The three leftmost columns show three representative velocity channels of the full cube centered on the [C II] emission. The rightmost column is the intensity-weighted velocity map. The top row is the data, whereas the remaining four rows shows the four different models described in Section 4.1. The velocity map for the four models is only shown in those pixels that have a $3 \sigma$ integrated flux density in the data.

further discussed in Section 5. All of the models have a decreasing intensity profile dependent only on the distance from the center. This clearly is an oversimplification, as the moment 0 map shows obvious evidence of nonexponential emission (see Figure 1).
The number of parameters in each of the models varies from 9 to 12 (Table 1). Each model contains the central position of the galaxy (in two spatial and one frequency dimension), the position angle of the maximum rotation, the central intensity and scale height of the emission, the maximum velocity of rotation, and the dispersion along the line of sight. Besides these eight parameters, the disk models also need an inclination, and disk models $\mathrm{B}$ and $\mathrm{C}$ require a scale radius for the velocity. Finally, the jet model $D$ requires three additional angles to describe the jet angle and opening angle of the jet, as well as a terminal radius at which the jet stops accelerating/ionizing the ISM.

From the fits shown in Figure 5, it is clear that the kinematics are inconsistent with a simple rotating disk with a constant velocity (Disk A), which is typically assumed in order to derive the dynamical masses of marginally resolved $z \sim 6$ quasar host galaxies (Wang et al. 2013; Venemans et al. 2016; Shao et al. 2017). To account for the extended emission detected at the systemic velocity, we need a truncated disk model (Disk C) whereby the systemic velocity goes to zero beyond a certain radius. Such a scenario could arise from either a gravitationally-dominated disk that has formed in the center of a dispersion-dominated source, or alternatively, a warp of the outer disk into the plane of the sky. Both scenarios, however, do not provide adequate fits to the observed [C II] distribution and kinematics (Figures 4 and 5).

As can be seen in Figure 4, from the position of the black hole, the velocity increases to a radius of $0.5 \mathrm{kpc}(0 . ! 1)$ and reaches a peak line-of-sight velocity of $150-200 \mathrm{~km} \mathrm{~s}^{-1}$. Assuming these kinematics can be described by a rotating disk, and the dynamical mass within this radius is $M_{\text {dyn }}=2.3 \times 10^{9} / \sin ^{2}(i) M_{\odot}$, with $i$ the inclination angle of the disk. For comparison, the mass of the black hole is $1 \times 10^{9} M_{\odot}$, and the inferred gas mass within this radius is $1.6 \times 10^{10} M_{\odot}$. For an inclination angle of $i=35^{\circ}$ (see Table 1), the dynamical mass and molecular gas mass within the central $1 \mathrm{kpc}$ are consistent with each other within the large uncertainties. Beyond this radius, the line-of-sight velocity is 
decreasing faster than Keplerian (shown by the Disk B model in Figure 4), and reaches approximately systemic velocity at the last measured points at $1.5-2.0 \mathrm{kpc}$. To summarize, the kinematics appear to be dispersion-dominated with some overall rotation (i.e., net angular momentum) in the central kiloparsec. This implies that most of the gas has not yet settled in a disk.

\section{Origin of the $[\mathrm{C} \mathrm{II}]$ Cavities}

Instead of a kinematic origin, the presence of the twokiloparsec-scale [C II] cavities could be the result of energy injection into the ISM. Similar shell-like structures are seen in the neutral ISM of local galaxies (e.g., Walter \& Brinks 1999), and are often interpreted as the results of supernova or AGN feedback. We can calculate the energy needed to created such structures seen here using (Chevalier 1974)

$$
E=5.3 \times 10^{43} n_{0}^{1.12}(d / 2)^{3.12} v_{\text {exp }}^{1.4} \operatorname{erg}
$$

with $n_{0}$ being the density in $\mathrm{cm}^{-3}, d$ being the diameter of the cavity in parsecs, and $v_{\exp }$ the expansion velocity in $\mathrm{km} \mathrm{s}^{-1}$. The cavities have a diameter of approximately $\sim 0.5 \mathrm{kpc}$ (see Figure 3), and the luminosity-weighted, average density of the ISM in the quasar host has been estimated to be $10^{5} \mathrm{~cm}^{-3}$ (Venemans et al. 2017) based on a multiline analysis in this source. Under the assumption that the S-like structure is due to energy input in the plane of the galaxy, the expansion velocity can be approximated by the amplitude of the derivation from systemic velocity (e.g., Walter \& Brinks 1999). We estimate an amplitude of $\sim 80 \mathrm{~km} \mathrm{~s}^{-1}$ at the center of the cavities (Figure 4). Taken together, we derive a required energy of $\sim 3 \times 10^{59} \mathrm{erg}$, or the equivalent of the mechanical energy output of 300 million typical SNe II (Woosley \& Weaver 1986). This large number of supernovae would have to be centrally clustered in both cavities or in between the cavities. If we assume that 1 supernova explodes for every 200 solar masses of new stars (e.g., Diehl et al. 2006), we expect 7 supernovae to form each year for the observed star formation rate of $1500 M_{\odot} \mathrm{yr}^{-1}$ (Venemans et al. 2016). The above total number of supernovae would then require a constant star formation rate over $\sim 40$ million years in a very restricted volume. However, from the dust continuum map (Figure 1) it is clear that the high star formation rate is distributed over a large, $5 \mathrm{kpc} \times 5 \mathrm{kpc}$ area, requiring an even longer period of high star formation activity in or between the cavities to inject enough energy into the ISM to create the cavities. On the other hand, the required energy can be easily produced by the central accreting supermassive black hole which has a bolometric luminosity of $L_{\text {bol }}=7.5 \times 10^{46} \mathrm{erg} \mathrm{s}^{-1} \quad$ (Mazzucchelli et al. 2017), or $2.4 \times 10^{54} \mathrm{erg} \mathrm{yr}^{-1}$. Assuming that $5 \%$ of the accreted energy is deposited as thermal energy in the ISM (e.g., Di Matteo et al. 2005), the required energy to create the cavities would be generated in only 2.5 million years, which is much shorter than the Salpeter time (or e-folding time) of the black hole of 45 million years (e.g., De Rosa et al. 2014; Mazzucchelli et al. 2017). If the black hole was indeed the cause of the cavities, the implied energy injection would be toward both sides, consistent with a simple jet-driven picture. This concept that holes in the gas distribution can be created by AGN jets is supported by numerical simulations (e.g., Gaibler et al. 2012). However, modeling of such a jet requires again a very unique distribution of the gas (Section 4.1). Regardless, the process needed to create these cavities does not suppress the star formation. This has also been seen in luminous quasars at $z \sim 2$ (e.g., Harrison et al. 2012) and predicted by some simulations (e.g., Gaibler et al. 2012).

\section{Companion Galaxies}

The environment of J0305-3150 has been the subject of various studies. A study searching for $\operatorname{Ly} \alpha$ emission from galaxies at the redshift of the quasar revealed the presence of a Ly $\alpha$ halo around the quasar, and a faint Ly $\alpha$ emitting (LAE) companion at a distance of $12.5 \mathrm{kpc}$ (Farina et al. 2017). In addition, a larger field-of-view narrowband search for Ly $\alpha$ emitters around the quasar indicated that the quasar is not located in a dense environment on megaparsec scales (Ota et al.

2018). Our previous, shallow ALMA data of the field contained two faint (rest frame $158 \mu \mathrm{m}$ flux densities of $S_{\text {cont }, 158 \mu \mathrm{m}} \ll 1 \mathrm{mJy}$ ) continuum sources (Venemans et al. 2016).

In addition to high-resolution imaging of the quasar host galaxy, the improved sensitivity of the new ALMA data allowed us to search for additional sources in the field. Previously, bright companion sources have been found in the vicinity of some high-redshift quasars (e.g., Decarli et al. 2017; Trakhtenbrot et al. 2017). Our new data reveal the presence of three [C II] emitters at the same redshift as the quasar (Figure 6; physical parameters in Table 2). One companion, $\mathrm{C} 1$, could already be seen in Figure 3. The brightest companion, C3, was already identified as a continuum source in the field in Venemans et al. (2016).

The FIR luminosity of companion $\mathrm{C} 3$ is comparable to that of the $[\mathrm{C} \mathrm{II}]$ companions near several $z \sim 6$ quasars presented in Decarli et al. (2017). However, in contrast to the companions found by Decarli et al. (2017), all companions identified near J0305-3150 have FIR luminosities that are a factor $\lesssim 10$ smaller than that of the quasar host. Also, the estimated molecular gas masses of the companions are at least a factor of $\sim 5$ smaller than that of the quasar host. Despite the small masses, the interaction of companions with the quasar host could provide an alternative explanation of the gas morphology and kinematics in the quasar host galaxy. Furthermore, it is a possibility that such interactions triggered the high star formation rate in the quasar host and the AGN activity.

\section{Summary}

The high spatial resolution $(\sim 400 \mathrm{pc})$ imaging of a quasar host galaxy at $z=6.6$ shows that its formation is a complex and chaotic process. We find that the ISM in the quasar host has not yet settled in a simple disk. While there are signs that the AGN is affecting the ISM, this feedback is not suppressing the formation of stars in the quasar host. In fact, the star formation rate inferred by the FIR luminosity of $\sim 1500 M_{\odot}$ $\mathrm{yr}^{-1}$ (Venemans et al. 2016) is high among galaxies and quasar hosts at similar redshifts (e.g., Carilli \& Walter 2013; Venemans et al. 2018). The observed high star formation rate and rapid black hole growth could be triggered by interactions with the newly detected nearby companion galaxies. The observations presented here show the unique role that high- 

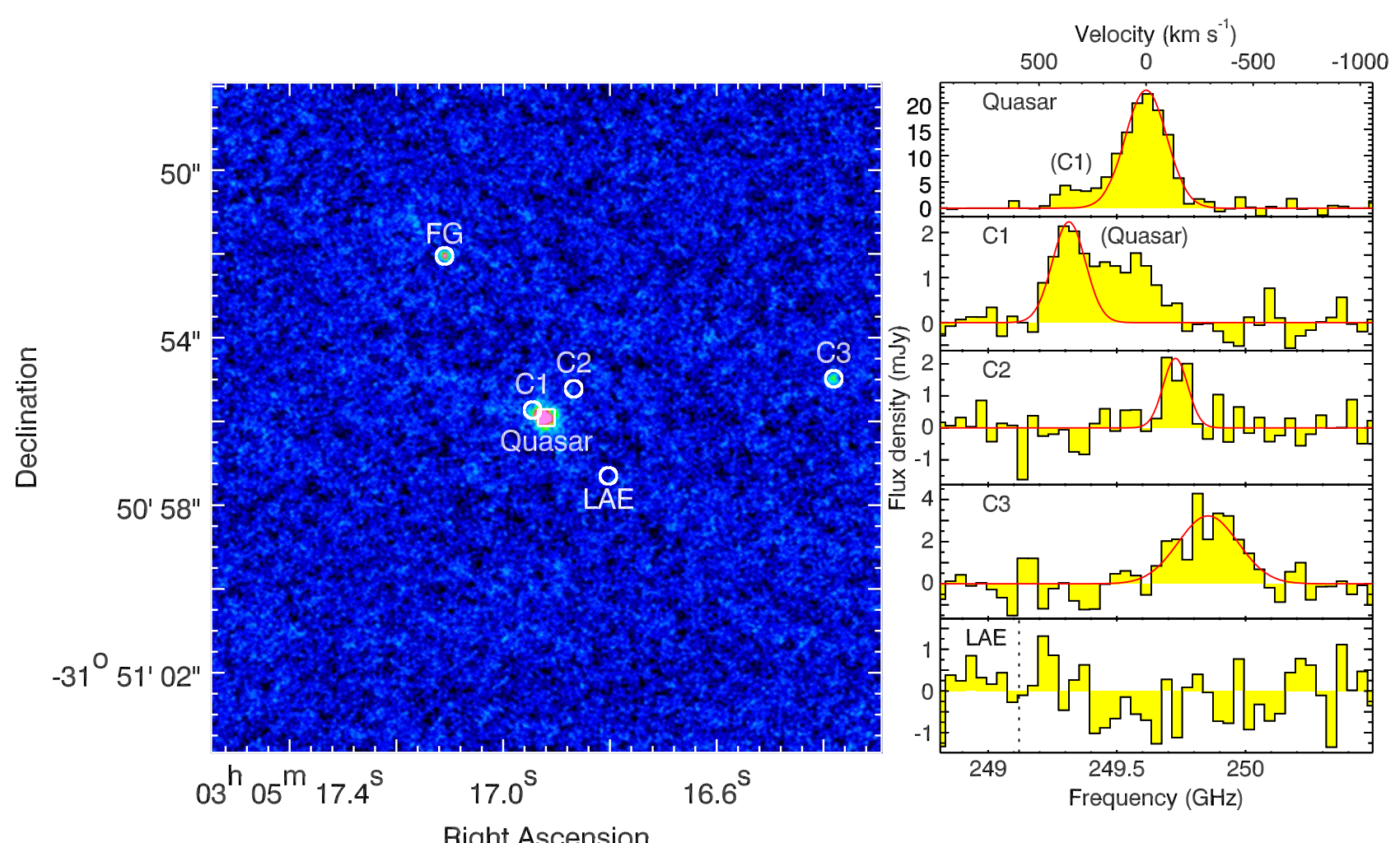

Figure 6. Left panel: continuum image of the field surrounding the quasar. This image size is $16^{\prime \prime} \times 16^{\prime \prime}\left(86.5 \times 86.5 \mathrm{kpc}^{2}\right)$. Several objects are detected near the quasar and are marked with a circle. Three [C II] emitting companions and a Ly $\alpha$ emitting galaxy (Farina et al. 2017) are located within $40 \mathrm{kpc}$ and $\sim 1000 \mathrm{~km} \mathrm{~s}{ }^{-1}$. The ALMA spectrum of the continuum source labeled FG did not reveal any emission lines. This source is likely not located near the quasar, but in the foreground. Right panel: spectra of the [C II] emitting companions and the quasar host galaxy. The characteristics of the companion galaxies are listed in Table 2. Due to the close proximity of $\mathrm{C} 1$ to the quasar host, the spectra of both $\mathrm{C} 1$ and the quasar host are overlapping. This is indicated in the spectra.

Table 2

Measured and Derived Properties of the Quasar and the Companion Galaxies

\begin{tabular}{|c|c|c|c|c|c|}
\hline Source: & Quasar & $\mathrm{C} 1$ & $\mathrm{C} 2$ & $\mathrm{C} 3$ & LAE \\
\hline R.A. & $03^{\mathrm{h}} 05^{\mathrm{m}} 16^{\mathrm{s}} .919$ & $03^{\mathrm{h}} 05^{\mathrm{m}} 16^{\mathrm{s}} .945$ & $03^{\mathrm{h}} 05^{\mathrm{m}} 16^{\mathrm{s}} .868$ & $03^{\mathrm{h}} 05^{\mathrm{m}} 16^{\mathrm{s}} \cdot 381$ & $03^{\mathrm{h}} 05^{\mathrm{m}} 16^{\mathrm{s}} .803$ \\
\hline Decl. & $-31^{\circ} 50^{\prime} 55^{\prime \prime} 901$ & $-31^{\circ} 50^{\prime} 55^{\prime \prime} 728$ & $-31^{\circ} 50^{\prime} 55^{\prime \prime} 220$ & $-31^{\circ} 50^{\prime} 54^{\prime \prime} 980$ & $-31^{\circ} 50^{\prime} 57^{\prime \prime} 300$ \\
\hline Redshift $^{\mathrm{a}}$ & $6.61391 \pm 0.00015$ & $6.6231 \pm 0.0003$ & $6.6104 \pm 0.0004$ & $6.6066 \pm 0.0006$ & $6.629 \pm 0.001$ \\
\hline Distance (kpc) & $\ldots$ & 2.0 & 5.1 & 37.4 & 11.0 \\
\hline$\Delta v_{\mathrm{los}}^{\mathrm{b}}\left(\mathrm{km} \mathrm{s}^{-1}\right)$ & $\ldots$ & +361 & -137 & -289 & +595 \\
\hline$F_{[\mathrm{C} \mathrm{II}]}^{\mathrm{a}}\left(\mathrm{Jy} \mathrm{km} \mathrm{s}^{-1}\right)$ & $5.43 \pm 0.33$ & $0.43 \pm 0.05$ & $0.31 \pm 0.08$ & $1.13 \pm 0.17$ & $<0.23$ \\
\hline $\mathrm{FWHM}_{[\mathrm{C} \mathrm{II}]}\left(\mathrm{km} \mathrm{s}^{-1}\right)$ & $225 \pm 15$ & $180 \pm 25$ & $135 \pm 40$ & $330 \pm 55$ & - \\
\hline$S_{\text {cont }, 158 \mu \mathrm{m}^{\mathrm{c}}}(\mathrm{mJy})$ & $5.34 \pm 0.19$ & $\ldots^{\mathrm{d}}$ & $<0.23$ & $0.58 \pm 0.12$ & $<0.33$ \\
\hline$L_{[\mathrm{C} \mathrm{II]}}\left(L_{\odot}\right)$ & $(5.9 \pm 0.4) \times 10^{9}$ & $(4.7 \pm 0.5) \times 10^{8}$ & $(3.4 \pm 0.8) \times 10^{8}$ & $(1.2 \pm 0.2) \times 10^{9}$ & $<2.5 \times 10^{8}$ \\
\hline$L_{\mathrm{FIR}}\left(L_{\odot}\right)$ & $(1.60 \pm 0.06) \times 10^{13}$ & $\ldots$ & $<5.2 \times 10^{11}$ & $(1.3 \pm 0.3) \times 10^{12}$ & $<7.7 \times 10^{11}$ \\
\hline $\operatorname{SFR}_{[\mathrm{C} \mathrm{II}]}^{\mathrm{e}}\left(M_{\odot} \mathrm{yr}^{-1}\right)$ & $1016 \pm 73$ & $51 \pm 9$ & $35 \pm 10$ & $159 \pm 28$ & $<25$ \\
\hline$M_{\mathrm{H}_{2}}^{\mathrm{f}}\left(M_{\odot}\right)$ & $(1.8 \pm 0.1) \times 10^{11}$ & $(1.5 \pm 0.2) \times 10^{10}$ & $(1.1 \pm 0.2) \times 10^{10}$ & $(3.7 \pm 0.6) \times 10^{10}$ & $<7.8 \times 10^{9}$ \\
\hline
\end{tabular}

Notes. All the quoted errors are $1 \sigma$ and the upper limits are $3 \sigma$.

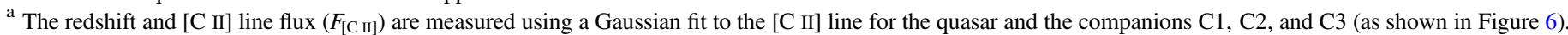
The redshift of the Ly $\alpha$ emitter (LAE) is taken from Farina et al. (2017).

${ }^{\mathrm{b}}$ The line-of-sight velocity $v_{\text {los }}$ is computed using $v_{\text {los }}=\left(z_{\text {companion }}-z_{\text {quasar }}\right) /\left(1+z_{\text {quasar }}\right) \times c$.

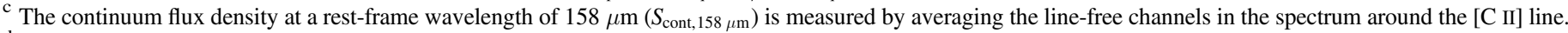

d The continuum flux density of $\mathrm{C} 1$ could not be accurately determined due to contamination by the quasar host galaxy.

e The star formation rate $\mathrm{SFR}_{[\mathrm{C} \text { II] }}$ is derived using $\mathrm{SFR}_{[\mathrm{C} \mathrm{II}]}=3 \times 10^{-9} L_{[\mathrm{C} \mathrm{II}]}^{1.18}$ (De Looze et al. 2014).

${ }^{\mathrm{f}}$ Molecular gas mass, derived from the [C II] luminosity using a $L_{[\mathrm{C} \mathrm{II}]}$-to- $\mathrm{H}_{2}$ conversion factor of $\alpha_{[\mathrm{C} \mathrm{II}]}=31 M_{\odot} / L_{\odot}(\mathrm{Zanella}$ et al. 2018).

angular resolution observations with ALMA can play in studies of the ISM in some of the most distant massive galaxies.

B.P.V., M.N., and F.W. acknowledge funding through the ERC grant "Cosmic Gas." This paper makes use of the following ALMA data: ADS/JAO.ALMA\#2017.1.01532.S. ALMA is a partnership of ESO (representing its member states), NSF (USA) and NINS (Japan), together with NRC (Canada), and NSC and ASIAA (Taiwan), in cooperation with 
the Republic of Chile. The Joint ALMA Observatory is operated by ESO, AUI/NRAO, and NAOJ.

Facility: ALMA.

\section{ORCID iDs}

Bram P. Venemans (iD https://orcid.org/0000-0001-9024-8322

Marcel Neeleman (iD https://orcid.org/0000-0002-9838-8191

Fabian Walter (iD https://orcid.org/0000-0003-4793-7880

Roberto Decarli (iD https://orcid.org/0000-0002-2662-8803

Joseph F. Hennawi (i) https://orcid.org/0000-0002-7054-4332

Hans-Walter Rix (ib https://orcid.org/0000-0003-4996-9069

\section{References}

Bañados, E., Venemans, B. P., Mazzucchelli, C., et al. 2018, Natur, 553, 473 Beelen, A., Cox, P., Benford, D. J., et al. 2006, ApJ, 642, 694

Bertoldi, F., Cox, P., Neri, R., et al. 2003, A\&A, 409, L47

Capak, P. L., Carilli, C., Jones, G., et al. 2015, Natur, 522, 455

Carilli, C. L., \& Walter, F. 2013, ARA\&A, 51, 105

Chevalier, R. A. 1974, ApJ, 188, 501

Decarli, R., Walter, F., Venemans, B. P., et al. 2017, Natur, 545, 457

Decarli, R., Walter, F., Venemans, B. P., et al. 2018, ApJ, 854, 97

De Looze, I., Cormier, D., Lebouteiller, V., et al. 2014, A\&A, 568, A62

De Rosa, G., Venemans, B. P., Decarli, R., et al. 2014, ApJ, 790, 145

Diehl, R., Halloin, H., Kretschmer, K., et al. 2006, Natur, 439, 45

Di Matteo, T., Croft, R. A. C., Feng, Y., Waters, D., \& Wilkins, S. 2017, MNRAS, 467, 4243

Di Matteo, T., Springel, V., \& Hernquist, L. 2005, Natur, 433, 604

Farina, E. P., Venemans, B. P., Decarli, R., et al. 2017, ApJ, 848, 78

Foreman-Mackey, D., Hogg, D. W., Lang, D., \& Goodman, J. 2013, PASP, 125,306

Gaia Collaboration, Brown, A. G. A., Vallenari, A., et al. 2018, A\&A, 616, A1
Gaibler, V., Khochfar, S., Krause, M., \& Silk, J. 2012, MNRAS, 425, 438

Gullberg, B., Swinbank, A. M., Smail, I., et al. 2018, ApJ, 859, 12

Harrison, C. M., Alexander, D. M., Mullaney, J. R., et al. 2012, ApJL, 760, L15

Helou, G., Khan, I. R., Malek, L., \& Boehmer, L. 1988, ApJS, 68, 151

Herrera-Camus, R., Sturm, E., Graciá-Carpio, J., et al. 2018, ApJ, 861, 95

Maiolino, R., Cox, P., Caselli, P., et al. 2005, A\&A, 440, L51

Mazzucchelli, C., Bañados, E., Venemans, B. P., et al. 2017, ApJ, 849, 91

McMullin, J. P., Waters, B., Schiebel, D., Young, W., \& Golap, K. 2007, ASP Conf. Ser. 376, Astronomical Data Analysis Software and Systems XVI, ed. R. A. Shaw, F. Hill, \& D. J. Bell (San Francisco, CA: ASP), 127

Mortlock, D. J., Warren, S. J., Venemans, B. P., et al. 2011, Natur, 474, 616 Ota, K., Venemans, B. P., Taniguchi, Y., et al. 2018, ApJ, 856, 109

Planck Collaboration, Ade, P. A. R., Aghanim, N., et al. 2016, A\&A, 594, A13 Sandstrom, K. M., Leroy, A. K., Walter, F., et al. 2013, ApJ, 777, 5

Shao, Y., Wang, R., Jones, G. C., et al. 2017, ApJ, 845, 138

Smith, J. D. T., Croxall, K., Draine, B., et al. 2017, ApJ, 834, 5

Trakhtenbrot, B., Lira, P., Netzer, H., et al. 2017, ApJ, 836, 8

Valiante, R., Schneider, R., Salvadori, S., \& Gallerani, S. 2014, MNRAS, 444, 2442

Venemans, B. P., Decarli, R., Walter, F., et al. 2018, ApJ, 866, 159

Venemans, B. P., Findlay, J. R., Sutherland, W. J., et al. 2013, ApJ, 779, 24

Venemans, B. P., McMahon, R. G., Walter, F., et al. 2012, ApJL, 751, L25

Venemans, B. P., Walter, F., Decarli, R., et al. 2017, ApJ, 845, 154

Venemans, B. P., Walter, F., Zschaechner, L., et al. 2016, ApJ, 816, 37

Walter, F., Bertoldi, F., Carilli, C., et al. 2003, Natur, 424, 406

Walter, F., \& Brinks, E. 1999, AJ, 118, 273

Walter, F., Carilli, C., Bertoldi, F., et al. 2004, ApJL, 615, L17

Wang, R., Carilli, C. L., Neri, R., et al. 2010, ApJ, 714, 699

Wang, R., Carilli, C. L., Wagg, J., et al. 2008, ApJ, 687, 848

Wang, R., Wagg, J., Carilli, C. L., et al. 2013, ApJ, 773, 44

Willott, C. J., Bergeron, J., \& Omont, A. 2015, ApJ, 801, 123

Willott, C. J., Omont, A., \& Bergeron, J. 2013, ApJ, 770, 13

Woosley, S. E., \& Weaver, T. A. 1986, ARA\&A, 24, 205

Zanella, A., Daddi, E., Magdis, G., et al. 2018, MNRAS, 481, 1976 